\title{
EXPRESSION PATTERNS OF THE STIM1 PROTEIN DURING DIFFERENT STAGES OF MOUSE TOOTH DEVELOPMENT
}

\author{
Walid El-Sayed*
}

\begin{abstract}
Objectives: Stromal interaction molecule 1 (STIM1), a calcium sensor protein, was shown to be significantly up-regulated during the maturation stage of amelogenesis. This result highlighted a potentially important role for STIM1 in calcium ion handling during the latter stages of enamel formation. Our work aimed to study in detail the expression patterns of STIM1 during all stages of mouse tooth development.

Material and Methods: C57BL mice were used for all the experiments in this study. The embryos were obtained from time mated pregnant mice. Embryonic day 0 (E0) was designated as the day on which a vaginal plug was confirmed. Embryos at E12.5, E14.5, E15.5 and pups at PNO, PN4, PN8 and PN14 were used to describe different developmental stages of incisors (E12.5 E15.5) and molars (PNO - PN14).

Results: Stim1 signals start to be detectable in the cells of the enamel organ at the beginning of the cap stage. Its expression was similar between the incisor and molar tooth germs. Odontoblasts appeared completely negative, while there was some strong expression in the middle of the dental papilla.

Conclusion: In our study, we have shown that Stim1 was most highly expressed in the ameloblasts during the maturation stage. This highly expression of Stim1- at the maturation stages of enamel development supports the contention that stored operated calcium channels are central to $\mathrm{Ca}^{+2}$ transport/extrusion mechanism during the final phase of enamel crystal growth.
\end{abstract}

KEYWORDS: STIM1, Tooth Development, Mineralisation, Calcium ion.

\section{INTRODUCTION}

Mammalian teeth are heterodont (i.e. they have differing shapes: incisors, canines, premolars and molars) that usually are replaced only once during the lifetime ${ }^{1}$. Development of the mammalian teeth is a complex process that results from sequential and reciprocal interaction between oral ectodermal and the underlying mesenchymal tissues of the first branchial $\mathrm{arch}^{2}$. This interaction is completely under the genetic control of many signaling molecule ${ }^{3}$.

\footnotetext{
* College of Oral and Dental Surgery, Oral Biology Department, Sues Canal University- Ismailia, Egypt.
} 
These signalling molecules have been shown to be secreted first by the oral epithelium and then to diffuse into the underlying ectomesenchyme inducing expression of a group of transcription factors $^{4}$. This reciprocal interaction between these signalling molecules and the corresponding transcription factors regulates and controls the morphodifferentiation and cytodifferentiation of different cells of the tooth germ ${ }^{5}$. As a result of this continuous interaction, a tooth germ is formed that includes the enamel organ, dental papilla and dental follicle. The tooth germ goes through different morphological stages until the tooth is completely formed ${ }^{6}$. First, the oral ectoderm thickens to form the primary epithelial band that gives rise to the dental lamina. The dental lamina subsequently invaginates into the ectomesenchyme corresponding to the positions of the future teeth. It then proceeds to form bud, cap and finally bell shape structures ${ }^{2,3,6}$. At the late bell stage of the tooth development, ameloblasts, specialised epithelial derived cells of the enamel organ, differentiate and start to secrete extracellular matrix which later will be mineralised to form dental enamel. Mature dental enamel is acellular, nonvital, and without capacity for turnover or significant repair ${ }^{7}$. Amelogenesis, the process by which enamel is produced, is divided mainly into secretory stage and maturation stages. During the secretory stage, the columnar-shaped ameloblasts secrete a protein-rich, partially mineralised, self-organising enamel matrix. ${ }^{8}$ This matrix is mainly composed of a group of extracellular enamel matrix (ECM) proteins, including three main structural proteins amelogenin (AMELX MIM*300391), enamelin (ENAM; MIM *606585), and ameloblastin (AMBN MIM *601259) and two enamel proteases, matrix metallopeptidase-20 (MMP20; MIM *604629]) and kallikrein 4 (KLK4; MIM *603767). When the matrix reaches full thickness, the secretory stage ends and the ameloblasts enter the maturation stage. The main function of the maturation stage ameloblasts is to harden the enamel by removal of the organic material from the enamel matrix and the secretion of more calcium and phosphates ions7. The amount of the secreted calcium ions during the maturation stage is estimated to be four-fold more than that secreted in the secretory stage ${ }^{8,9}$. However, how the ameloblasts control and handle this great amount of mineral ions, especially calcium, is still not clear and requires more investigation. However, how the ameloblasts control and handle this great amount of mineral ions, especially calcium, is still not clear and requires more investigation.

Stromal interaction molecule 1 (STIM1), a calcium sensor protein, was shown to be significantly up-regulated during the maturation stage of amelogenesis ${ }^{10}$. This result highlighted a potentially important role for STIM1 in calcium ion handling during the latter stages of enamel formation. Our work aimed to study in detail the expression patterns of STIM1 during all stages of mouse tooth development.

\section{MATERIALS AND METHODS}

\section{Animals}

C57BL mice were used for all the experiments in this study. Adult C57BL mice were housed in a temperature controlled room under artificial illumination with access to food and water ad libitum (Clinical Science Building, St. James's University Hospital-Leeds University-UK). The embryos were obtained from time mated pregnant mice. Embryonic day 0 (E0) was designated as the day on which a vaginal plug was confirmed. Embryos at E12.5, E14.5, E15.5 and pups at PNO, PN4, PN8 and PN14 were used to describe different developmental stages of incisors (E12.5 - E15.5) and molars (PN0 - PN14). The work was carried out in accordance with The Code of Ethics of the World Medical Association (Declaration of Helsinki).

\section{Sections preparation and immune-staining}

Immediately after sacrifice of the pregnant 
mother at the right embryological time, whole embryos were fixed with $4 \%$ paraformaldehyde (PFA) in $0.01 \mathrm{M}$ phosphate buffered saline (PBS, $\mathrm{pH}$ 7.4), processed and embedded in paraffin using standard conditions. Heads of puppies at PN4, PN8 and PN14 were decalcified in $8 \%$ formic acids for two weeks before they were processed and embedded in paraffin. Serial sections were taken from all the paraffin blocks at a thickness of $5 \mu \mathrm{m}$ and a selection stained with Haematoxylin and eosin $(H \& E)$ to obtain correct orientation of the different developmental stages of the tooth bud. Selected sections were used for Stim1 immunoe-staining as follows; Sections were de-waxed in two changes of xylene for 20 minutes each, and then placed in 2 changes of absolute alcohol for 5 minutes each. The endogenous peroxidase activity was blocked by immersing the slides in $2 \%$ hydrogen peroxide/ methanol solution for 10 minutes. Monoclonal Stim1 primary antibody was obtained commercially (Sigma-UK), loaded at 1/200 dilution and incubated for 1 hour at room temperature. EnVision kits were then used according to the manufacturer's instructions (Dako EnVision K5007).

\section{RESULTS}

\section{Expression pattern of Stim1 during early stages of mouse incisor development}

The tooth germ started to be developmentally clear at E12.5 (Figure 1). Stim1 expression was not clear at this stage in any of the cells of the enamel organ or dental papilla. However, Stim1 expression was very clear in the alveolar bone surrounding (Figure 1). At E14.5, the tooth germ entered the cap stage where all components of the enamel organ [outer enamel (OEE), stellate reticulum (SR) and inner enamel epithelium (IEE)] became very clearly delineated (Figure 1). During this sage, Stim1 expression was evident in the inner enamel epithelium, especially the enamel knot but was still very weakly expressed in the rest of the enamel organ. The dental papilla was almost free of any expression. Stim1 signal was still clear and strong in the alveolar bone surrounding the Meckel's cartilage and in the Meckel's cartilage cells themselves (Figure 1).

At E15.5, the tooth germ proceeded to very early bell stage where the incisor tooth morphology was determined. Stratum intermedium (SI) became very clear, located as two layers of stratified epithelium just above the IEE cells (Figure 1, IIIC). The onset of odontoblast differentiation became evident. Expression of Stim1 was localised at this stage to the IEE cells of the enamel organ, especially on the labial side and incisal third of the lingual side of the developing incisor (Figure 1, IIIB). Expression of Stim1 also was strong in SI, OEE and SR cells of the enamel organ. Odontoblasts appeared completely negative, while there was some strong expression in the middle of the dental papilla. Alveolar bone surrounding the tooth germ showed strong expression of Stim1.

\section{Expression pattern of Stim1 during mouse lower molar development}

Development of mouse molars is not continuous throughout the life of the animal, so it is more akin to human teeth development. In this study Stim1 expression was traced and localized in sections of lower molars at embryonic days PN0, PN4, PN8 and PN14 as these times present a complete range of the different stages of enamel formation. At PNO, the molar tooth germ appeared to be at late early bell stage, where all cells of the enamel organ could be easily seen and the multi-cusp nature of the tooth was evident (Figure 2, IA). Stim1 expression pattern was similar to that seen in the incisor at a similar stage (Figure 1, IIIB and C) where the expression was localized to the IEE, SR and, to a lesser extent, OEE cells. Odontoblasts were completely negative at this stage (Figure 2, IC). 
At PN4, the ameloblasts and the rest of the enamel organ were in pre-secretory or early secretory stage of enamel formation. Stim1 expression was clear but weak in the ameloblasts and in the remainder of the enamel organ (Figure 2, IIC). At PN8 all the ameloblasts had reached the secretory stage of enamel formation. Stim1 was localised in ameloblasts and the papillary layer of the enamel organ. The expression pattern was similar to that seen at PN4. However at PN14 where ameloblasts in the cuspal regions were at the more advanced, maturation stage of enamel development, Stim1 was highly expressed in these cells (Figure 2, IVB and C).

Columns I, II and III show the mouse lower incisor at bud, cap, and early bell stages respectively. Rows A\&B show these different developmental stages stained with $H \& E$ and with Stim1 primary antibodies respectively. Rows $\mathrm{C}$ and $\mathrm{D}$ show higher magnification views of row B. Stim1 expression was not detected in the cells of the enamel organ (EO) or dental papilla (DP) at the bud stage (I.C), but was localized mainly in the centre part of the inner enamel epithelium (IEE) (enamel knot) (black arrow) of the enamel organ at the cap stage (II.C) and was expressed in all the cells of the enamel organ at the early bell stage (III.C). Stim1 was expressed in the alveolar bone $(\mathrm{AB})$ surrounding the developing tooth from the bud to early bell stages (Row D). Its expression was not clear in Meckel's cartilage (MC) at the bud stage (I.E), faint at the cap stage (II.E) and strong at the early bell stage (III.E). OEE = outer enamel epithelium, IEE = inner enamel epithelium, $\mathrm{SR}=$ stellate reticulum, $\mathrm{SI}=$ stratum intermedium, $\mathrm{MC}=$ Meckel's cartilage, $\mathrm{A}=$ ameloblasts and $\mathrm{O}=$ odontoblasts.

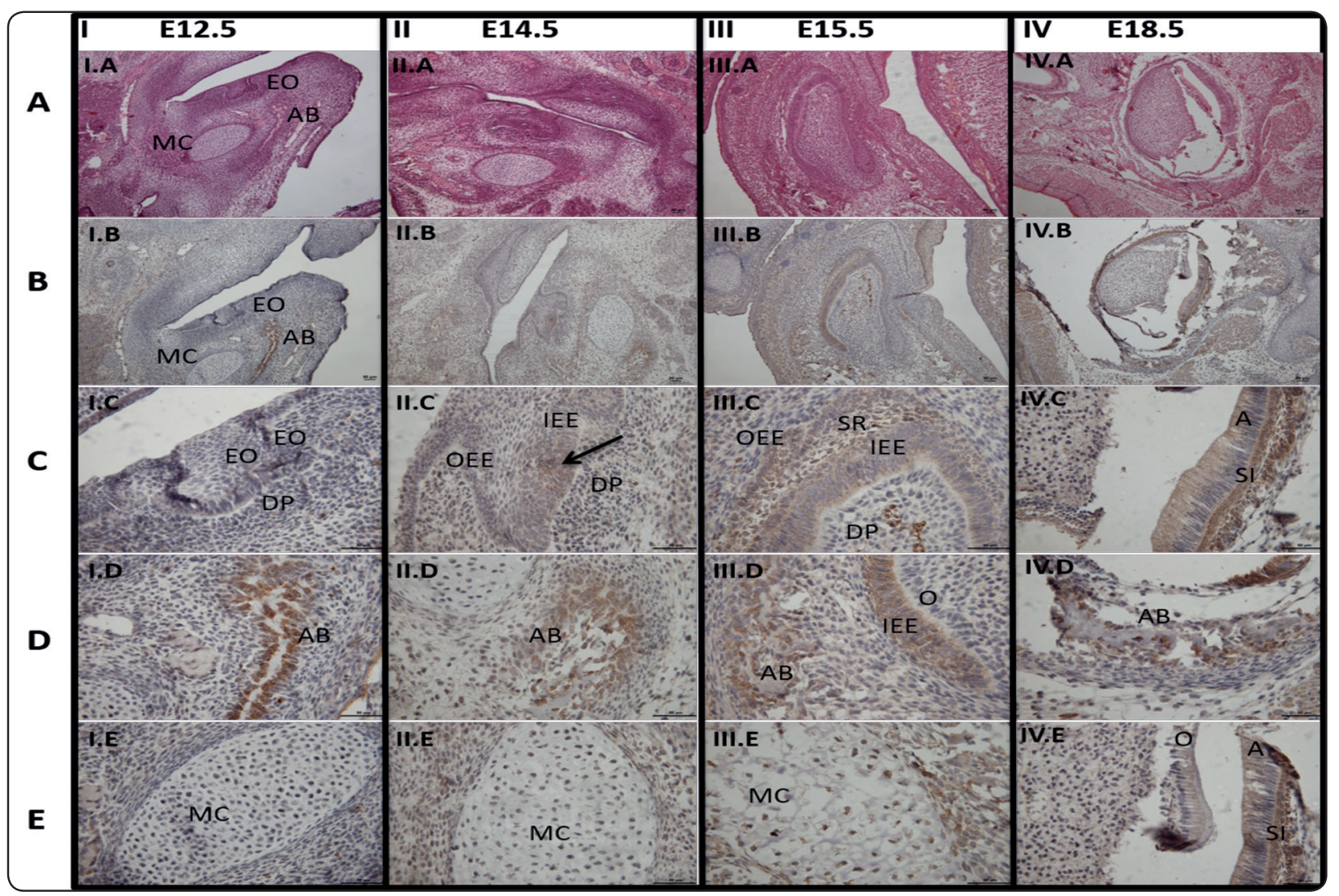

Fig. (1) Expression patterns of Stim1 in mouse lower incisor development 
Columns I, II, III and VI show developmental stages of mouse lower molars at cap, presecretory, secretory, transition and maturation stages respectively. Rows A\&B show these stages stained with H\&E and with Stim1 primary antibodies respectively. At PNO, Stim1 expression was localized to the inner enamel epithelium (IEE), stratum intermedium (SI) and stellate reticulum (SR) of the enamel organ but not the dental papilla (DP), or the oral epithelium (OE) (I.B and C). At PN4, Stim1 expression appeared in the cytoplasm of ameloblasts (A) and odontoblasts (O) (II.C). The expression intensity of Stim1 was stronger, however at PN8 (III C) and significantly stronger at PN14 (IV.C), localized mainly in the cytoplasm of maturation stage ameloblasts. $\mathrm{PL}=$ papillary layer.

\section{DISCUSSION}

In this study, we have investigated the expression pattern of Stim1 protein during tooth development. Stim1 signals start to be detectable in the cells of the enamel organ at the beginning of the cap stage. Its expression was similar between the incisor and molar tooth germs when germs at the same developmental stage were examined. Stim1 expression was not restricted to any one type of cell of the tooth germ, but was localised in most of them to a greater or lesser extent at some point in the development cycle. By far the most significant expression was in maturation stage ameloblasts.

STIM1 is a type I transmembrane protein with an EF-hand motif near the amino terminus, and is located in the lumen of the endoplasmic reticulum

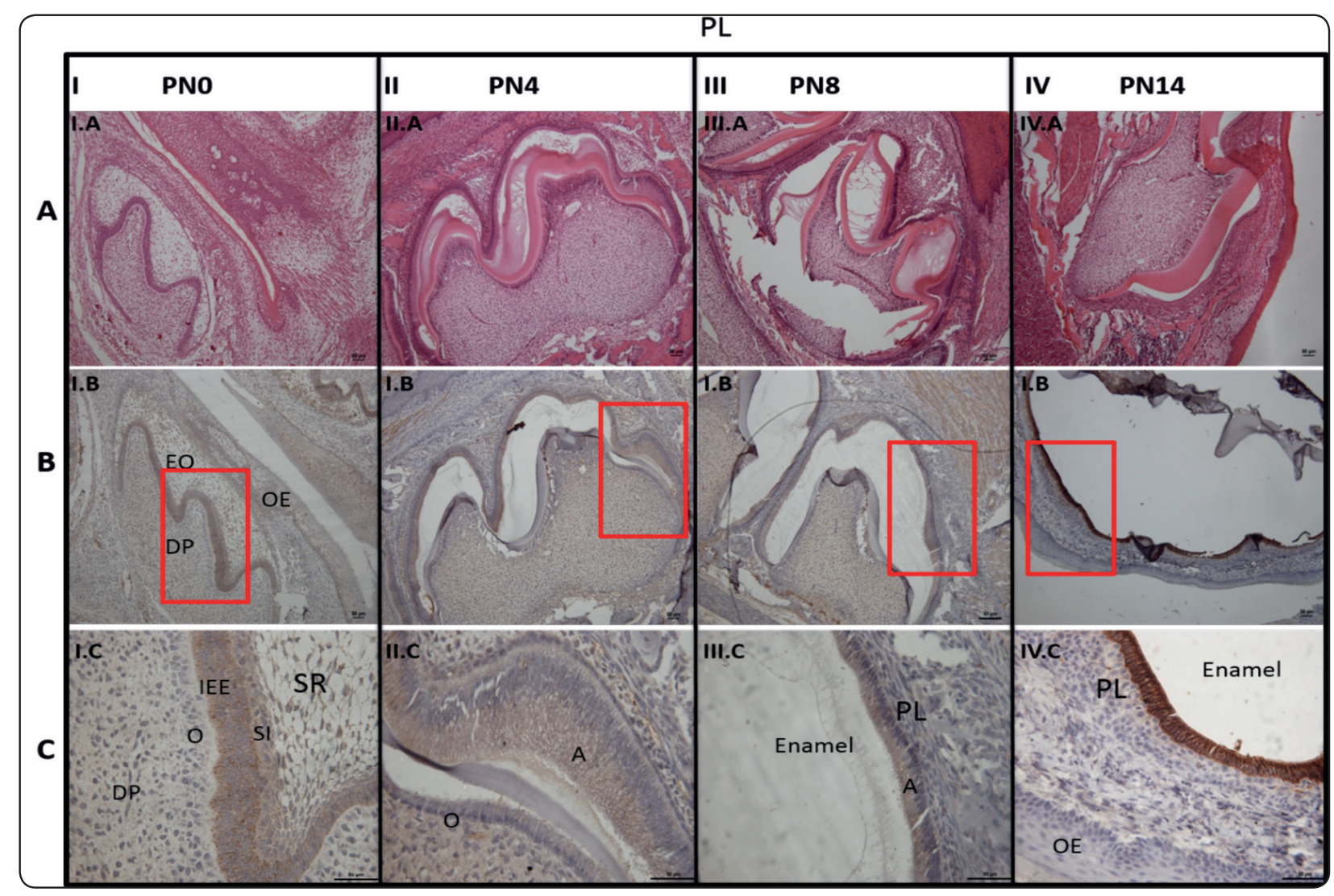

Fig. (2) Expression patterns of Stim1 in mouse lower molars development. 
$(E R)^{11}$. Many studies have localised Stim1 to the membrane of the endoplasmic reticulum, where it acts as a $\mathrm{Ca}^{+2}$-ion sensor inside the lumen through its EF- hand motif. Stim1, together with members of the ORAI family of proteins (ORAI 1, ORAI 2 and ORAI 3), are subunits of store- operated calcium channels $(\mathrm{SOC})^{12,13}$. In the resting state of the cell, $\mathrm{Ca}^{2+}$ ions inside the lumen of ER bind the EF- hand motif of STIM1, and keep the SOC channels closed. Depletion of $\mathrm{Ca}^{2+}$ ions from the ER lumen, results in a release of $\mathrm{Ca}^{2+}$ ions from the EF-hand motif of STIM1, leading to conformational changes and oligomerisation of STIM1. These changes lead to rapid translocation of STIM1 to form clusters immediately adjacent to the plasma membrane (PM) at ER-PM junctions, bringing the endoplasmic reticulum (ER) near to the plasma memberane. STIM1 interacts with ORAI proteins resulting in activation and opening of SOC channels. Activation of SOC channels leads to rapid influx of $\mathrm{Ca}^{2+}$ ions in to the $\mathrm{ER}^{13 ; 14}$. Calcium ions $\left(\mathrm{Ca}^{2+}\right)$ are very important for enamel formation, as they constitute the main mineral element. Despite its importance, the $\mathrm{Ca}^{2+}$ transport/extrusion mechanism in ameloblasts during enamel formation remains to be fully elucidated.

The $\mathrm{Ca}^{2+}$ transport/extrusion mechanism can be divided into three stages: $\mathrm{Ca}^{2+}$ entry, transit through the cell cytoplasm or organelles and finally extrusion through the plasma membrane ${ }^{9,15} \cdot \mathrm{Ca}^{2+}$ ions pass through channels that can be classified into voltage gated $\mathrm{Ca}^{2+}$ channels which open and close due to changes in the membrane potential and nonvoltage gated $\mathrm{Ca}^{2+}$ channels ${ }^{14}$. Store-operated $\mathrm{Ca}^{2+}$ (SOC) are included in the latter. Once $\mathrm{Ca}^{2+}$ ions enter, there are two scenarios; they either bind to the cytoplasmic binding proteins and remain there until secreted ( calcium ferry dogma), or enter the intracellular $\mathrm{Ca}^{2+}$ stores such as the endoplasmic reticulum (ER) and mitochondria prior to be secreted through the plasma membrane by exocytosis 9,15 .
Most recent studies tend to argue against the calcium ferry dogma as they suggest that ameloblasts in the maturation stage actually down-regulate most of the cytoplasmic calcium binding proteins ${ }^{16}$. At the same time, it was found that the maturation stage ameloblasts up-regulate the whole group of proteins related to the intracellular $\mathrm{Ca}^{2+}$-stores, including endoplasmic reticulum $\mathrm{Ca}^{2+}$ binding proteins (calreticulin and endoplasmin), endoplasmic reticulum $\mathrm{Ca}^{2+}$ - ATPase, and Inositol 1,4,5-trisphosphate receptors ${ }^{9}$. The above data may support the idea that the maturation stage ameloblasts may rely on the intracellular $\mathrm{Ca}^{2+}$ stores such as the endoplasmic reticulum in the $\mathrm{Ca}^{2+}$ efflux, thus highlighting the importance and the role of Stim1 in this process. Developing enamel in the maturation stage requires a large amount of free calcium ions for supporting the rapid growth of the hydroxyapatite crystals in both width and thickness ${ }^{9}$. It was demonstrated that efflux of calcium ions through ameloblasts is 3-4 fold higher in the maturation stage than in the secretion stage ${ }^{8}$. In our study, we have shown that Stim1 was most highly expressed in the ameloblasts during the maturation stage. This result agrees with a recent study which demonstrated that Stim1 was up-regulated during the late stage of enamel formation using real time $\mathrm{PCR}^{10}$. This difference in the expression of Stim-1 between secretory and maturation stages of enamel development supports the contention that stored operated calcium channels are central to $\mathrm{Ca}^{2+}$ transport/extrusion mechanism during the final phase of enamel crystal growth.

\section{REFERENCES}

1. Moustakas JE, Smith KK, Hlusko LJ. Evolution and development of the mammalian dentition: insights from the marsupial Monodelphis domestica. Dev Dyn2011; 240(1):232-9.

2. Thesleff I. Epithelial-mesenchymal signalling regulating tooth morphogenesis. J Cell Sci2003; 116(Pt 9) 116: 16471648 . 
3. Tompkins K. Molecular mechanisms of cytodifferentiation in mammalian tooth development. Connect Tissue Res2006; 47(3):111-8.

4. Bei M. Molecular genetics of ameloblast cell lineage. J Exp Zoolog B Mol Dev Evol2009; 312B (5): 437-444.

5. Tummers M. and Thesleff I. The importance of signal pathway modulation in all aspects of tooth development. $\mathrm{J}$ Exp Zool B Mol Dev Evol2009; 312B(4): 309-319.

6. Rothová M, Peterková R, Tucker AS. Fate map of the dental mesenchyme: dynamic development of the dental papilla and follicle. Dev Biol2012; 366(2):244-54.

7. Simmer JP, Papagerakis P, Smith CE, Fisher DC, Rountrey AN, Zheng L, et al. Regulation of dental enamel shape and hardness. J Dent Res2010; 89(10):1024-38.

8. Smith C.E. Cellular and chemical events during enamel maturation. Crit Rev Oral Biol Med1998; 9(2): 128-161.

9. Hubbard M. J. Calcium transport across the dental enamel epithelium. Crit Rev Oral Biol Med2000; 11(4): 437-466.

10. Lacruz RS, Smith CE, Bringas P Jr, Chen YB, Smith SM, Snead ML, et al. Identification of novel candidate genes involved in mineralization of dental enamel by genome-wide transcript profiling. J Cell Physiol2012; 227(5): 2264-2275.
11. Sabbioni S, Barbanti-Brodano G, Croce CM, Negrini M. GOK: a gene at 11p15 involved in rhabdomyosarcoma and rhabdoid tumor development. Cancer Res1997;57(20): 4493-4497.

12. Giachini FR, Webb RC, Tostes RC. STIM and Orai proteins: players in sexual differences in hypertension-associated vascular dysfunction? Clin Sci (Lond); 118(6): 391-396.

13. Zhou Y, Meraner P, Kwon HT, Machnes D, Oh-hora M, Zimmer J, et al. STIM1 gates the store-operated calcium channel ORAI1 in vitro. Nat Struct Mol Biol2010; 17(1): 112-116.

14. Cahalan M. D. Cell biology. How to STIMulate calcium channels. Science2010; 330(6000): 43-44.

15. Hubbard, M. J. Abundant calcium homeostasis machinery in rat dental enamel cells. Up-regulation of calcium store proteins during enamel mineralization implicates the endoplasmic reticulum in calcium transcytosis. Eur J Biochem1996; 239(3): 611-623.

16. Hubbard MJ, McHugh NJ, Mangum JE. Exclusion of all three calbindins from a calcium-ferry role in rat enamel cells. Eur J Oral Sci2011; 119 Suppl 1: 112-119. 